EDITORA

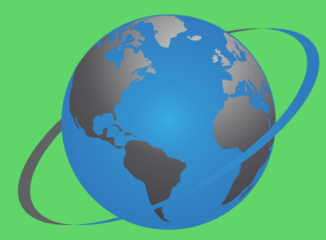

OMNIS SCIENTIA

\title{
SAÚDE PÚBLICA EM
}

\section{TEMPOS PANDEMICOS}

\section{VOLUME 1}

Organizador:

Raul Sousa Andreza

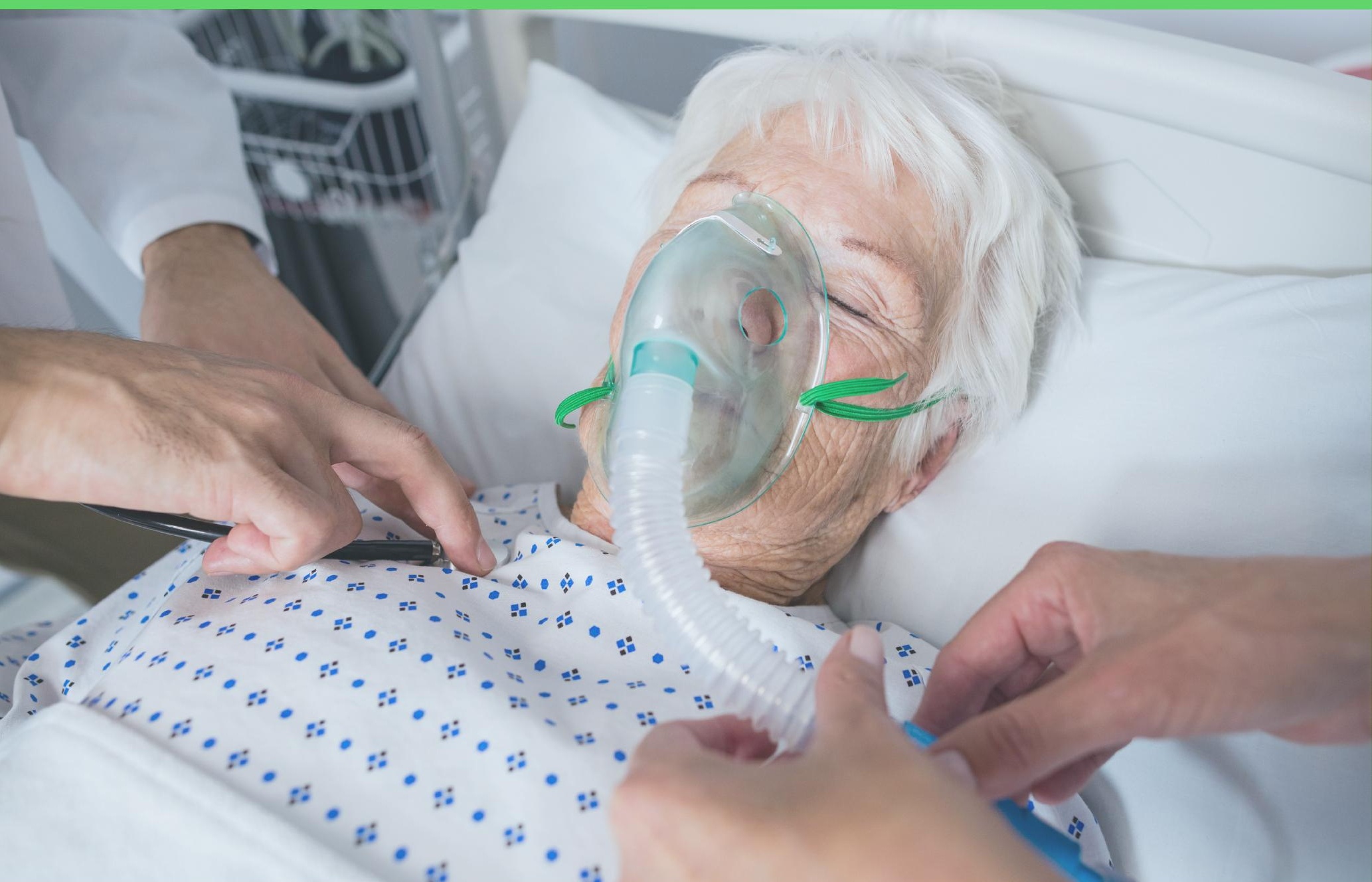


EDITORA

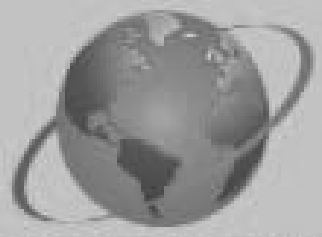

OMNIS SCIENTIA

\section{SAÚDEE PÚBUICA BM}

\section{THEMIPOS PANDENMICOS}

\section{VOLUME 1}

Organizador:

Raul Sousa Andreza

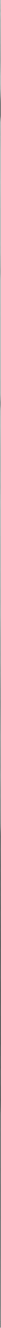


Editora Omnis Scientia

SAÚDE PÚBLICA EM TEMPOS PANDÊMICOS

Volume 1

$1^{a}$ Edição

TRIUNFO - PE

2021 


\section{Editor-Chefe}

Me. Daniel Luís Viana Cruz

Organizador (a)

Prof. Me. Raul Sousa Andreza

\section{Conselho Editorial}

Dra. Pauliana Valéria Machado Galvão

Dr. Wendel José Teles Pontes

Dr. Walter Santos Evangelista Júnior

Dr. Cássio Brancaleone

Dr. Plínio Pereira Gomes Júnior

Editores de Área - Ciências da Saúde

Dra. Camyla Rocha de Carvalho Guedine

Dra. Cristieli Sérgio de Menezes Oliveira

Dr. Leandro dos Santos

Dr. Hugo Barbosa do Nascimento

Dr. Marcio Luiz Lima Taga

Dra. Pauliana Valéria Machado Galvão

\section{Assistentes Editoriais}

Thialla Larangeira Amorim

Andrea Telino Gomes

Imagem de Capa

Freepik

\section{Edição de Arte}

Vileide Vitória Laranjeira Amorim

\section{Revisão}

Os autores

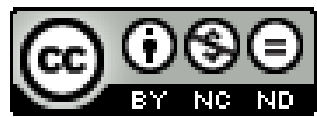

Este trabalho está licenciado com uma Licença Creative Commons - AtribuiçãoNãoComercial-SemDerivações 4.0 Internacional.

O conteúdo abordado nos artigos, seus dados em sua forma, correção e confiabilidade são de responsabilidade exclusiva dos autores. 
Dados Internacionais de Catalogação na Publicação (CIP) (eDOC BRASIL, Belo Horizonte/MG)

S255 Saúde pública em tempos pandêmicos [livro eletrônico] / Organizador Raul Sousa Andreza. - Triunfo, PE: Omnis Scientia, 2021.

286 p. : il.

Formato: PDF

Requisitos de sistema: Adobe Acrobat Reader

Modo de acesso: World Wide Web

Inclui bibliografia

ISBN 978-65-88958-41-4

DOI 10.47094/978-65-88958-41-4

1. Ciências da saúde. 2. Saúde pública. 3. Pandemia. I. Andreza, Raul Sousa.

CDD 610

Elaborado por Maurício Amormino Júnior - CRB6/2422

\author{
Editora Omnis Scientia \\ Triunfo - Pernambuco - Brasil \\ Telefone: +55 (87) 99656-3565 \\ editoraomnisscientia.com.br \\ contato@editoraomnisscientia.com.br
}




\section{PREFÁCIO}

A incidência do novo coronavírus no Brasil é preocupante. No entanto, a saúde pública do país e o sistema de atendimento visa abranger a diversidade que o Brasil apresenta, o sistema único de saúde (SUS) tem como base a integralidade, a universalidade e a equidade de todos os pacientes e trabalhadores. Instituído assim, para democratizar toda a saúde brasileira, tem o interesse de ofertar serviços de qualidade a população. Portanto, ao longo da história de sua consolidação a saúde pública foi deixado de lado e passou a gerar grandes problemas aos profissionais atuantes.

De fato, os estudos desenvolvidos no âmbito da saúde pública se propõem a articular conhecimentos de diferentes saberes e fazeres fornecendo subsídios teóricos, práticos e metodológicos que contribuem positivamente para a construção de estratégias e políticas públicas que visam o desenvolvimento de informações e ações em prol de uma saúde de qualidade para toda comunidade.

O presente livro é composto por 24 capítulos elaborados por autores pertencentes às ciências da saúde e suas áreas afins com o objetivo de proporcionar conhecimentos e compartilhar experiências e resultados de estudos desenvolvidos em várias localidades brasileiras e que visam à elucidação de diferentes situações de saúde.

Em nossos livros selecionamos um dos capítulos para premiação como forma de incentivo para os autores, e entre os excelentes trabalhos selecionados para compor este livro, o premiado foi o capítulo 02, intitulado "VACINAS CONTRA COVID-19: UMA BREVE DESCRIÇÃO POR MEIO DE REVISÃO INTEGRATIVA“. 


\section{SUMÁRIO}

CAPÍTULO 1

A IMPORTÂNCIA E OS DESAFIOS DA ESTRATÉGIA DE SAÚdE DA FAMÍLIA NO ENFRENTAMENTO DA COVID-19

Romana Erica Tavares Grangeiro Pinto

Wyara Ferreira Melo

Maria Amanda Laurentino Freires

Patrício Borges Maracajá

Aline Carla de Medeiros

José Cândido da Silva Nóbrega

Manoel Marques de Souto Nóbrega Filho

Túlio Alberto de Oliveira Sousa

Mônica Valéria Barros Pereira

Vicente Saraiva dos Santos Neto

Hozanna Estrela Celeste

Gabriela Rocha Pordeus dos Santos

DOI: 10.47094/978-65-88958-41-4/17-26

CAPÍTULO 2

VACINAS CONTRA COVID-19: UMA BREVE DESCRIÇÃO POR MEIO DE REVISÃO INTEGRATIVA

Fernanda Lima Marçal

Isabela Figueiredo e Souza

Maria Eduarda Coelho Gomes

Larissa Lima Torres

Isabela Campbell Santos

Thamara Lóren Lima

Ludmilla Vieira Magalhães

Maria Eduarda Sirina Pereira

Lucas Viana de Oliveira 
Larissa da Silva Torres França

Natan Fiorotti da Silva

Milena de Oliveira Simões

DOI: 10.47094/978-65-88958-41-4/27-41

CAPÍTULO 3

AÇÃO EM SAÚdE E A IMPORTÂNCIA DO USO CORRETO DAS MÁSCARAS PARA PREVENÇÃO DE INFECÇÕES RESPIRATÓRIAS

Soniely Nunes de Melo

Rafael Belarmino de Souza Lima

Tarcísio Correia Sposito

Rayana Ribeiro Trajano de Assis

Nayara Sandriele Santana de Souza

Bruna Rafaella Santos Torres

Flávio José Alencar de Melo

Davi Silva de Jesus

Carlos Henrique Bezerra de Siqueira

Izabelle Barbosa da Silva

Marcos André de Holanda Prudente Pessoa

Ana Marlusia Alves Bomfim

DOI: 10.47094/978-65-88958-41-4/42-51

CAPÍTULO 4

CONHECIMENTOS DAS EQUIPES MULTIPROFISSIONAIS DA ESTRATÉGIA DE SAÚDE DA FAMIILIA SOBRE COVID-19 E GESTAÇÃO

Priscilla dos Santos Nascimento

Michelle Araújo Moreira

DOI: 10.47094/978-65-88958-41-4/52-65 
CARTILHA EDUCATIVA SOBRE A COVID-19 PARA A PROTEÇÃO DA COMUNIDADE ACADÊMICA DO CAMPUS BELÉM DO IFPA

Lidineusa Machado Araujo

Maria de Nazaré Pereira Rodrigue Martins

Gabriela Priscila de Lima Carvalho

Fernanda Rafaela de Souza Rebelo da Costa

Michelle da Silva Pereira

Andréa de Melo Valente

Maria Helena Cunha Oliveira

Antônio Marcos Mota Miranda

DOI: 10.47094/978-65-88958-41-4/66-75

CAPÍTULO 6 .76

A SAÚdE DOS PROFISSIONAIS DE SAÚDE QUE ATUAM NA LINHA DE FRENTE NA PANDEMIA DO COVID - 19: REVISÃO INTEGRATIVA

João Lucas Ferreira Andrade

Léa Bianch Lima

Luana Kellen Nogueira Epitácio

Maria Eduarda Alves Vasconcelos

Antônio Augusto Ferreira Carioca

Carlos Antônio Bruno da Silva

Eudóxia Sousa de Alencar

DOI: 10.47094/978-65-88958-41-4/76-89

CAPÍTULO 7 .90

OS EFEITOS DA PANDEMIA NA COMPULSÃO ALIMENTAR: UMA REVISÃO DE LITERATURA

Lais Pontes de Miranda Cerqueira

Tarcio Goncalves sobral

Isadora Bianco Cardoso de Menezes

DOI: 10.47094/978-65-88958-41-4/90-97 
O IMPACTO DA PANDEMIA DO COVID - 19 SOBRE A SEGURANÇA ALIMENTAR E NUTRICIONAL: REVISÃO INTEGRATIVA

Bruna Sousa Barbosa

Igor Matheus Cruz de Oliveira

João Lucas Ferreira Andrade

Léa Bianch Lima

Luana Kellen Nogueira Epitácio

Maria Eduarda Alves Vasconcelos

Antônio Augusto Ferreira Carioca

Carlos Antônio Bruno da Silva

Eudóxia Sousa de Alencar

DOI: 10.47094/978-65-88958-41-4/98-104

CAPÍTULO 9 105

A UTILIZAÇÃO DOS PROTOCOLOS DE USO DO GUIA ALIMENTAR PARA A POPULAÇÃO BRASILEIRA: RELATO DE EXPERIÊNCIA

Bárbara Santos Amorim

Lis Chaves Marinho

Isadora Bianco Cardoso de Menezes

DOI: 10.47094/978-65-88958-41-4/105-114

CAPÍTULO 10 .

O IMPACTO DA COVID-19 EM PORTADORES DE SÍNDROME METABÓLICA

Laura Rasul de Lima

Ana Beatriz Amaral Vieira

Gabriella Neiva Reis

Ingrid Ravenna Liberalino Lima

DOI: 10.47094/978-65-88958-41-4/115-122 
SIMPLIFICANDO A HANSENÍASE: PROJETO DE INTERVENÇÃO REALIZADO EM

MEIO DIGITAL DURANTE A PANDEMIA

Amanda Almeida Lima

Ana Beatriz Sousa Santos

Francisco Vittor Miranda e Araújo

Jesamar Correia Matos Filho

João Ferreira de Paula Neto

Maria Clara de Freita Albano

Manoel Cícero Viana de Lima

Pedro Schmitt Martins Paiva Matos

Ruddy Mariano Maia Cysne Guerra

Samuel Carvalho Vasconcelos

Thaine Mirla Rocha

Elaine Lopes Bomfim

DOI: 10.47094/978-65-88958-41-4/123-131

CAPÍTULO 12.

DESCOMPLICANDO A HANSENÍASE: PROJETO DE INTERVENÇÃO REALIZADO EM UNIDADE DE SAÚDE NO PERÍODO DA PANDEMIA

Adália Stefanny de Araujo Cavalcante

Giovanna Giffoni Souza do Nascimento

Iêda de Freitas Martins Jota

Isabel Camila Araújo Barroso

Kaio Rangel Freitas Guimarães

Laís Mesquita de Sousa

Monique dos Santos Chaves

Manoel Victor Freires Vieira

Matheus Macedo Braga Coelho

Thaine Mirla Rocha

Elaine Lopes Bomfim 
CAPÍTULO 13. 140

HANSENÍASE: UM ESTUDO EPIDEMIOLÓGICO NA REGIÃO DO MÉDIO ARAGUAIAMATO GROSSO

Flavia Rodrigues Santana

Josilene Dália Alves

DOI: 10.47094/978-65-88958-41-4/140-150

CAPÍTULO 14 151

MUNICÍPIOS COM ALTA INCIDÊNCIA DE TUBERCULOSE EM MATO GROSSO: CONHECER PARA INTERVIR EM TEMPOS DE PANDEMIA

Larissa Machado Bellé

Yasmim Paloma Abreu Silva

Alessandro Rolim Scholze

Josilene Dália Alves

DOI: 10.47094/978-65-88958-41-4/151-162

CAPÍTULO 15 163

SAÚDE INTEGRAL DA MULHER EM CONTEXTO PANDÊMICO: REVISÃO INTEGRATIVA DE LITERATURA

Lohana Guimarães Souza

Tailande Venceslau Carneiro

Letícia Grazielle Santos

DOI: 10.47094/978-65-88958-41-4/163-175

CAPÍTULO 16 176

ASSISTÊNCIA DE ENFERMAGEM NO OUTUBRO ROSA E PERFIL EPIDEMIOLÓGICO DAS MULHERES: RELATO DE EXPERIÊNCIA

Helena Pereira de Souza

Laura Letícia Perdição Guerra

Luana Fernandes e Silva 
Thales Philipe Rodrigues da Silva

Alessandra Lage Faria

Helen Carine Ferreira Balena

Érica Moreira de Souza

Bruna Luíza Soares Pinheiro

Lorena Medeiros de Almeida Mateus

Flávia Duarte de Oliveira Ribeito

Bianca Maria Oliveira Luvisaro

Fernanda Penido Matozinhos

DOI: 10.47094/978-65-88958-41-4/176-188

CAPÍTULO 17 189

AGRAVAMENTO DAS DOENÇAS PSIQUIÁTRICAS DURANTE O PERÍODO DE ISOLAMENTO SOCIAL: UMA BREVE REVISÃO DE LITERATURA

Hellen Kristina Magalhães Brito

Gabriela Teixeira Lima

Ana Laura Fernandes Tosta

Laura Beatriz Caitano de Oliveira

Maria Paula Ricardo Silva

Mariana Vieira Garcia de Carvalho

Nathália Siriano Costa

Mayara Rita Figueredo

Mabel Fernandes Rocha

Helena Maria Mendes Marques

Kaio Murilo Santana Corrêa

Ana Flávia Buiatte Andrade

DOI: 10.47094/978-65-88958-41-4/189-200 
ATUAÇÃO DO PSICÓLOGO EM TEMPO DE PANDEMIA: UMA REVISÃO DE ESTUDOS NACIONAIS

Gabriel Rigamonte

Sueli Souza

Wilson Quiroz

Daniel Bartholomeu

Fernando Pessotto

Cintia Heloína Bueno

Fernanda Helena Viana Garcia

DOI: 10.47094/978-65-88958-41-4/201-213

CAPÍTULO 19

CRIAÇÃO DE UM APLICATIVO VOLTADO PARA UMA UNIDADE BÁSICA DE SAÚdE EM FORTALEZA

Isabella Araujo Duarte

Giovanna Rolim Pinheiro Lima

Idna Lara Goes de Sena

Laura Figueiredo Leite

Letícia Cavalcante Lócio

Livian Araújo Camelo Gomes

Maria Regina Cardoso Linhares Oliveira Lima

Maria Tereza Linhares Cardoso

Pedro Henrique Cardoso Nogueira

Rafael Albuquerque Franco

Rodrigo Carvalho Paiva

Berta Augusta Faraday Sousa Pinheiro

DOI: 10.47094/978-65-88958-41-4/214-229 
TELEMEDICINA E SUAS VARIÁVEIS NO CENÁRIO DE PANDEMIA MUNDIAL: UMA REVISÃO DE LITERATURA

Raniere Canteiro Garcia Lhamas

Andressa Marcolino Campos

Douglas Ferreira Lima

Gabriel Souza Ferreira Oliveira

Guilherme de Mendonça Lopes Beltrão

Luciana de Paula Santana

Nícollas Nunes Rabelo

DOI: 10.47094/978-65-88958-41-4/230-237

CAPÍTULO 21 238

MONITORIA ACADÊMICA NO ENSINO REMOTO: PERSPECTIVAS E DESAFIOS EM TEMPOS PANDÊMICOS

Felipe Gabriel Assunção Cruz

Givanildo Bezerra de Oliveira

Marcílio Delan Baliza Fernandes

Ana Lúcia Moreno Amor

DOI: 10.47094/978-65-88958-41-4/238-248

CAPÍTULO 22

A DOENÇA DO OLHO SECO NA SÍINDROME DE SJÖGREN

Bruna Rafaella Santos Torres

Carlos Eduardo Ximenes da Cunha

Carlos Henrique Bezerra de Siqueira

Flavia Emanuelly Alves França Gomes

Santília Tavares Ribeiro de Castro e Silva

Anna Caroline Guimarães Gomes

Laís Rytholz Castro

Dennis Cavalcanti Ribeiro Filho 
Lara Medeiros Pirauá de Brito

Marina Viegas Rezende Ribeiro

DOI: 10.47094/978-65-88958-41-4/249-259

CAPÍTULO 23 260

FEIRAAGROECOLÓGICA: DIFICULDADES E POTENCIALIDADES DE UM CIRCUITO CURTO DE COMERCIALIZAÇÃO

Maria Rita Garcia de Medeiros

Rônisson Thomas de Oliveira Silva

Maria Natalícia de Lima

Ana Beatriz Macêdo Venâncio dos Santos

DOI: 10.47094/978-65-88958-41-4/260-269

CAPÍTULO 24 270

AVALIAÇÃO DO IMPACTO DE UMA REDE SOCIAL EM USUÁRIOS DE UMA UNIDADE BÁSICA DE SAÚDE EM FORTALEZA

Luiz Gerson Gonçalves Neto

Letícia Cavalcante Lócio

Carlos Alexandre Leite Pereira Filho

Henrique Sousa Costa

Maria Helena dos Santos Macedo

Lígia Bringel Olinda Alencar

Berta Augusta Faraday Sousa Pinheiro

Isaac Dantas Sales Pimentel

DOI: 10.47094/978-65-88958-41-4/270-280 


\section{CAPÍTULO 7}

OS EFEITOS DA PANDEMIA NA COMPULSÃO ALIMENTAR: UMA REVISÃO

DE LITERATURA

\section{Lais Pontes de Miranda Cerqueira ${ }^{1}$;}

Centro Universitario Cesmac, Maceió, Alagoas.

http://lattes.cnpq.br/0961379772375855

\section{Tarcio Goncalves sobral²;}

Centro Universitario Cesmac, Maceió, Alagoas.

http://lattes.cnpq.br/1467048286609188

Isadora Bianco Cardoso de Menezes ${ }^{3}$.

Centro Universitario Cesmac, Maceió, Alagoas.

http://lattes.cnpq.br/3284848999812706

RESUMO: A pandemia por COVID-19 trouxe grandes repercussões no cotidiano da população mundial, devido à necessidade de quarentena, isolamento e distanciamento social. O isolamento social imposto pela necessidade de restringir a transmissão do vírus conduziu as populações a conseguiram fazer adaptações negativas nos hábitos alimentares. O objetivo do estudo foi abordar a compulsão alimentar relacionada à restrição social e as mudanças nos estilos de vida, consequente da pandemia da COVID-19. A metodologia aplicada foi uma revisão de literatura de artigos publicados nos idiomas português, inglês e espanhol no período entre 2020 a 2021. A pandemia ocasionada pelo COVID-19 tem correlação direta com modificações nos hábitos alimentares dos indivíduos. Durante este período introduziu-se diversos estressores, incluindo solidão decorrente do isolamento social, medo de contrair a doença, tensão econômica e incerteza sobre o futuro. Observa-se que os sintomas apontados acerca da questão pandêmica atual, podem tornar-se maiores e mais relevantes, o que leva aos indivíduos uma atenção especial nos cuidados de saúde mental, visto que sua situação de vulnerabilidade se torna ainda mais complexa. Considerações: Esta pesquisa consistiu em uma revisão da literatura, de modo que pesquisas adicionais sobre a temática são essenciais. Nesse sentido, sugerem-se levantamentos sobre implicações na saúde mental diante da pandemia e sobre possíveis intervenções.

PALAVRAS-CHAVE: COVID 19. Compulsão alimentar. Ansiedade. 
ABSTRACT: The COVID-19 pandemic brought great repercussions on the daily lives of the world population, due to the need of quarantine, isolation and social distance. The social isolation imposed by the need for to decrease the transmission of the virus led populations to make positive changes in eating habits. The objective of this study was to address binge eating disorder related to social restriction and changes in lifestyles resulting from the COVID-19 pandemic. The applied methodology was a literature review of articles published in Portuguese, English and Spanish in the period between 2020 and 2021. The pandemic caused by COVID-19 has a direct correlation with changes in the eating habits of individuals. During this period, several stressors were introduced, including loneliness due to social isolation, fear of contracting the disease, economic tension and uncertainty about the future Conclusion: It is observed that the symptoms pointed out about the current pandemic issue, may become greater and more relevant, which gives individuals a special attention in mental health care, since their situation of vulnerability becomes even more complex. This research consisted of a literature review, so that further research on the subject is essential. In this sense, surveys on implications for mental health in the face of the pandemic and on possible interventions are suggested.

KEY-WORDS: COVID-19. Eating compulsion. Anxiety.

\section{INTRODUÇÃO}

Classificada como pandemia em março de 2020 pela Organização Mundial de saúde A COVID 19, até o momento conta com 15.282.705 casos na população do Brasil, sendo considerado o segundo pais com maior número de casos confirmados. Como formas protetivas diversas foram às medidas tomadas, visando controlar sua disseminação tendo em vista que seu mecanismo de transmissão se dá pelo ar e aglomeração (MALTA et al., 2020).

Como inicialmente o isolamento enfatizava a população idosa, grandes perdas vivenciadas e inúmeros casos de depressão surgiram com a população em questão e também o cancelamento das aulas, o que levou a um grande problema de socialização entre os jovens e adolescentes. Consequentemente, a busca por satisfação de outras formas para compensar toda a ansiedade tem resultado em graves problemas de saúde, refletindo diretamente nas condições de segurança alimentar (AQUINO et al., 2020).

Vale destacar que o ato de comer está ligado, para muitas pessoas, a uma forma de aliviar os sentimentos ruins como raiva ou até mesmo frustrações e sofrimentos. Porém este ato alimentar vai além, já que o corpo precisa de todas as necessidades nutricionais, que são fundamentais para o bom funcionamento do nosso organismo, para que assim também possamos prevenir doenças. A síndrome conhecida como Transtorno da Compulsão Alimentar Periódica (TCAP/DSM IV-TR) é a perda de controle sobre o quê e quanto se come, pelo menos dois dias por semana e não acompanhada de comportamentos compensatórios (WHO, 2021). 
O isolamento social imposto pela necessidade de restringir a transmissão do vírus conduziu as populações a conseguiram fazer adaptações positivas nos hábitos alimentares. Entretanto, o longo período de distanciamento levou alguns indivíduos pela ociosidade a beliscar entre as refeições, como uma maneira de suplantar a ansiedade vivenciada, hábito este, que é fator de risco para patologias como obesidade (WHO, 2021).

De acordo com dados da Organização Mundial de Saúde (OMS), quase 5\% da população do globo (aproximadamente 330 milhões de indivíduos) convivem com a depressão e as suas repercussões no cotidiano. Vale ressaltar que cada indivíduo reage ao isolamento e sente a situação de forma diferenciada.

Segundo o Manual Diagnóstico e Estatístico de Desordens Mentais (DSM-V, 2014), os transtornos alimentares são caracterizados por severas alterações no comportamento alimentar, podendo causar tanto a perda extrema, quanto o ganho excessivo de peso. A falta de controle que o paciente possui nas suas refeições, fazendo de forma rápida e exagerada, mesmo já estando saciado. A pandemia de COVID-19 introduziu diversos estressores, incluindo solidão decorrente do isolamento social, medo de contrair a doença, tensão econômica e incerteza sobre o futuro.

O presente estudo objetiva abordar a compulsão alimentar relacionada à restrição social e as mudanças nos estilos de vida consequente da pandemiada COVID-19.

\section{MATERIAS E METODOS}

A metodologia aplicada foi uma revisão literatura de artigos publicados nos idiomas português, inglês e espanhol, no período entre 2020 a 2021, obtidas nas plataformas: ScientificElectronic Library Online (SciELO), National Library of Medicine (PubMED) e o Google Acadêmico, tendo como as palavras chaves: COVID 19, Compulsão Alimentar, Ansiedade.

A amostra inicial com o uso dos descritores e qualificador na busca avançada foi de 190 estudos, que após a utilização dos filtros presentes nas bases (texto completo, idioma, ano, e tipo de arquivo), resultaram em 18 artigos para análise do título, sendo incluídos na revisão 07 artigos.

Não foram incluídos nesta revisão: livros, capítulos de livros, cartas aos editores, resenhas de livros e revistas não científicas (greyliterature). Também foram excluídos artigos não publicados no recorte temporal especificado (2020 a 2021) e que não estivessem disponíveis para download gratuito. Outros critérios de exclusão foram ausência dos descritores de busca no título ou nas palavras-chave do artigo e a publicação em periódicos com estrato B3, B4, B5 e C do Qualis CAPES.

\section{RESULTADOS E DISCUSSÕES}

Na pesquisa inicial nas bases de dados eletrônicas, foi realizado as combinações: compulsão alimentar e transtorno de compulsão alimentar; e ansiedade; identificando 125 artigos, sendo 88 publicados no período de 2020 a 2021 e nos idiomas descritos no método. Deste número, 18 estavam disponíveis na íntegra para download. Neste número parcial, identificamos que alguns artigos se 
repetiam uma ou mais vezes na mesma ou em diferentes bases de dados. Totalizando ao termino 7 artigos pertinentes ao tema publicados no ano de 2020 .

Quadro 1: Resultados dos artigos da revisão.

\begin{tabular}{|c|c|c|c|c|c|}
\hline Ano & Título & Autor & Método & Objetivo & Resultados \\
\hline 2020 & $\begin{array}{l}\text { A pandemia da } \\
\text { COVID-19 e as } \\
\text { mudanças no } \\
\text { estilo de vida } \\
\text { dos brasileiros } \\
\text { adultos: }\end{array}$ & $\begin{array}{l}\text { MALTA, Deborah } \\
\text { Carvalho } \\
\text { GRACIE, Renata }\end{array}$ & $\begin{array}{l}\text { E s t u d o } \\
\text { transversal }\end{array}$ & $\begin{array}{l}\text { Descrever as } \\
\text { mudanças nos } \\
\text { estilos de vida, } \\
\text { quanto ao consumo } \\
\text { de tabaco, bebidas } \\
\text { a } 1 \text { c o ó } 1 \text { i c a s, } \\
\text { alimentação e } \\
\text { atividade física, no } \\
\text { período de restrição } \\
\text { social consequente } \\
\text { à pandemia da } \\
\text { COVID-19. }\end{array}$ & $\begin{array}{l}\text { Durante o período de } \\
\text { restrição social, foi relatada } \\
\text { diminuição da prática de } \\
\text { atividade física e aumento } \\
\text { do tempo em frente a } \\
\text { telas, da ingestão de } \\
\text { alimentos ultraprocessados, } \\
\text { do número de cigarros } \\
\text { fumados e do consumo de } \\
\text { bebidas alcóolicas. Foram } \\
\text { observadas diferenças } \\
\text { segundo sexo e faixa etária. }\end{array}$ \\
\hline 2020 & $\begin{array}{lr}\text { Lazer e } & \text { Saúde } \\
\text { Mental } & \text { em } \\
\text { tempos } & \text { de } \\
\text { Covid-19 } & \end{array}$ & MENEZES, Suzy & $\begin{array}{l}\text { Revisão de } \\
\text { literatura }\end{array}$ & $\begin{array}{l}\text { Analisar como } \\
\text { saúde mental } \\
\text { e lazer foram } \\
\text { impactados durante } \\
\text { a pandemia por } \\
\text { COVID-19. }\end{array}$ & $\begin{array}{l}\text { A busca por estratégias } \\
\text { que atenuem os impactos } \\
\text { sobre a saúde mental e } \\
\text { sobreo lazer são essenciais } \\
\text { para lidar com a atual crise } \\
\text { devido à pandemia. }\end{array}$ \\
\hline 2020 & $\begin{array}{l}\text { T r a n s o r n o } \\
\text { de compulsão } \\
\text { a } 1 \text { i m e n t a r } \\
\text { periódica: uma } \\
\text { p e r s p e c t i v a } \\
\text { neurobiológica }\end{array}$ & $\begin{array}{l}\text { OLIVEIRA, Maria } \\
\text { Eduarda } \\
\text { CAVALCANTI, } \\
\text { Camilla }\end{array}$ & $\begin{array}{l}\text { Revisão de } \\
\text { literatura }\end{array}$ & $\begin{array}{l}\text { Sintetizar os dados } \\
\text { existentes sobre } \\
\text { os mecanismos } \\
\text { neurobiológicos } \\
\text { que caracterizam } \\
\text { o ranstorno } \\
\text { de } r \text { compulsão } \\
\text { alimentar periódica. }\end{array}$ & $\begin{array}{l}\text { Onão estabelecimento das } \\
\text { bases neurais características } \\
\text { do transtorno de compulsão } \\
\text { alimentar periódica } \\
\text { demonstra uma lacuna no } \\
\text { âmbito clínico, ressaltando } \\
\text { a necessidade de uma } \\
\text { investigaçãoneurobiológica } \\
\text { detalhada a fim da obtenção } \\
\text { de um diagnóstico mais } \\
\text { preciso. }\end{array}$ \\
\hline
\end{tabular}


Risco de possível a gravamento do transtorno o b s e s s i v o c o m puls i vo devido a pandemia covid-19
GOBBO, Ingrid; Revisão de Contribuir para Modos de enfrentamento, SILVIA, Veridiana; literatura análise e discussão bem como a maneira que BARSZCZ, Marcos acerca do risco de possível agravamento do transtorno obsessivo c o m p u $1 \mathrm{~s} \mathrm{i} \mathrm{v} \mathrm{o}$ devido à pandemia covid-19, bem como os aspectos p s i c o ló g i c o s envolvidos neste contexto e suas representações no cotidiano. a sociedade vem lidando com o isolamento. $\mathrm{O}$ autoconhecimento, a solidariedade, os olhares empáticos do outro despontam como práticas importantes para a promoção de saúde mental nesse contexto. ins.




de ansiedade e depressão. Estes indivíduos devem receber atenção especial nos cuidados de saúde mental, pois se encontram em situação de vulnerabilidade.

Ainda observando a relação entre fatores emocionais e os hábitos alimentares, em um estudo brasileiro, analisando dois grupos de indivíduos (um sem depressão, e o outro com diagnóstico prévio dessa patologia), pôde-se concluir: maior consumo de ultraprocessados $(17,6 \%$ e $24,6 \%$, respectivamente) e diminuição da ingesta de frutas e vegetais (78,1\% e $81,6 \%$, respectivamente) (CHOPRA 2020).

No que concerne a fatores pré-existentes como ativadores durante a pandemia, observou-se que indivíduos que apresentavam maiores preocupações com o corpo, também se mostraram com mais dificuldade na hora da aquisição alimentar (28\% da amostra), bem como maior insegurança pessoal (42,8\% da amostra) e maior prática de atividade física (58,9\% da amostra). Esses dados convergem para a conclusão de que o medo pelo COVID-19 influencia em aspectos psicológicos como a ansiedade e restrição alimentar, principalmente em indivíduos que já são propensos a essas características (HADDAD et al., 2020).

\section{CONCLUSÃO}

Observa-se que os sintomas apontados acerca da questão pandêmica atual, podem tornar-se maiores e mais relevantes, o que leva aos indivíduos uma atenção especial nos cuidados de saúde mental e a compulsão alimentar, visto que sua situação de vulnerabilidade se torna ainda mais complexa.

O presente estudo mostra, então, a necessidade de buscar melhorias para os costumes alimentares existentes na população, uma vez que muitos estão relacionados com o desenvolvimento de várias doenças (como obesidade, hipertensão, diabetes e entre outras).

\section{DECLARAÇÃO DE INTERESSES}

Nós, autores deste artigo, declaramos que não possuímos conflitos de interesses de ordem financeira, comercial, político, acadêmico e pessoal.

\section{REFERÊNCIAS}

American PsychiatricAssociation. (2014). Manual diagnóstico e estatístico de transtornos mentais DSM-5 (5a ed.). Porto Alegre: Artmed.

Aquino, Estela M. L. et al . Medidas de distanciamento social no controle da pandemia de COVID-19: potenciais impactos e desafios no Brasil. Ciênc. saúde coletiva,Rio de Janeiro , v. 25,supl. 1, p. 24232446, June 2020 . Available from.

Balodis, I. M.; Grilo, C. M.; Kober, H.; Worhunsky, P. D.; White, M. A.; Stevens, M. C.; Potenza, M. N. A pilot study linking reduced fronto-Striatal recruitment during reward processing to persistent 
bingeing following treatment for binge-eating disorder. International Journal of Eating Disorders, v. 47, n. 4, p. 376-384, 2014.

Bloc, Lucas Guimarães et al .Transtorno de compulsão alimentar: revisão sistemática da literatura. Rev. Psicol. Saúde, Campo Grande, v. 11,n. 1, p. 3-17,abr. 2019.

Brown, S. et al. A Qualitative Exploration of the Impact of COVID-19 on Individuals. Chopra, S. et al. Impact of COVID-19 on Lifestyle-Related Behaviours- a CrossSectional Audit of Responses from Nine Hundred and Ninety-Five Participants from India. Diabetes \&MetabolicSyndrome: ClinicalResearch\& Reviews. v. 14, n. 6, p. 2021-2030, Geliebter,

A., BENSON, L., PANTAZATOS, S. P., HIRSCH, J., CARNELL, S. Greater anterior cingulate activation and connectivity in response to visual and auditory high-calorie food cues in binge eating: Preliminary findings. Appetite, v. 96, p. 195-202, 2016.

Gobbo Ingrid; Silva Veridiana Soares da. Risco de possível agravamento do transtorno obsessivo compulsivo devido a pandemia - COVID-19 v. 18 n. 1 (2020): Discutindo o novo normal em tempos de pandemia.

Haddad, C. et al. Association Between Eating Behaviour and Quarantine/Confinement Stressors During the Coronavirus Disease 2019 Outbreak. J. of Eat. Disord. v. 8, n. 40, 2020.

Lima S. O., SilvaM. A. da, SantosM. L. D., MouraA. M. M., SalesL. G. D., MenezesL. H. S. de, NascimentoG. H. B., OliveiraC. C. da C., ReisF. P., \&JesusC. V. F. de. (2020). Impactos no comportamento e na saúde mental de grupos vulneráveis em época de enfrentamento da infecção COVID-19: revisão narrativa. Revista Eletrônica Acervo Saúde, (46), e4006.

Malta, Deborah Carvalho et al. A pandemia da COVID-19 e as mudanças no estilo de vida dos brasileiros adultos: um estudo transversal, 2020.Epidemiol. Serv. Saúde, Brasília , v. 29,n. 4, e2020407, set. 2020.

Menezes, S. K. de O. (2021). Lazer e Saúde Mental em Tempos de Covid-19. LICERE - Revista Do Programa De Pós-graduação Interdisciplinar Em Estudos Do Lazer, 24(1), 408-446.

Oliveira Maria Eduarda dos Santos Pereira de; Camilla de Andrade Tenorio Cavalcanti. Transtorno de compulsão alimentar periódica: uma perspectiva neurobiológicaBraz. J. ofDevelop., Curitiba, v. 6, n. 3, p. 10600-10607, mar. 2020. ISSN 2525-8761.

Pereira,MD, Oliveira, LC, Costa,CFT, Bezerra,CMO,Pereira,MD, Santos,CKA\& Dantas,EHM (2020). The COVID-19 pandemic, social isolation, consequences on mental health and coping strategies: an integrative review.Research, Society and Development, 9(7): 1-35, e652974548.

Vasconcelos, C. S.D.S. et al. O Novo Coronavírus e os Impactos Psicológicos da Quarentena. DESAFIOS-Revista Interdisciplinar da Universidade Federal do Tocantins, v. 7, n. Especial-3, p. 75-80, 2020.

With Eating Disorders in the UK. Appetite. v. 156, n. 2021, 2020. 
World Health Organization. WHO Coronavirus Disease (COVID-2019) situation reports. [Internet], jan. 2020. 


\section{Índice Remissivo}

$\mathbf{A}$

Acesso aos psicólogos 201, 203, 204

Acesso às redes sociais 43

Agricultor e o consumidor 260

Agricultores familiares 260, 263, 266

Alterações do metabolismo 115

Ambiente virtual 215

Ansiedade 77, 78, 81, 82, 83, 84, 86, 87, 91, 92, 94, 95, 117, 121, 190, 192, 193, 194, 197, 204, 207, 208, 209

Aplicativo 136, 215, 217, 218, 219, 220, 221, 222, 223, 224, 225, 226, 227, 228, 243, 271, 272, 273, 274, 278, 280

Aprendizagem 239

Assistência social 163, 171, 205

Associação da covid-19 com a gestação 52

Astrazeneca (universidade de oxford) 28, 30

Atenção primária 25, 124

Atenção primária à saúde (aps) 19, 59, 107, 125, 177, 178

Atendimento ambulatorial 105

Atendimento on-line 201, 203

Atendimento remoto 202, 205

Atividades econômicas 28

Autoanticorpos 250

Autoridades sanitárias 67, 69, 73, 278

C

Capacidade de defesa do organismo 115

Características dos imunizantes 28, 30

Cartilha educativa sobre a covid-19 67, 69, 70

Ceratoconjuntivite sicca $(\mathrm{kcs}) 250$

Combate à hanseníase 133

Comercialização 38, 260, 261, 262, 263, 264, 265, 266, 267, 268, 269

Compulsão alimentar 90, 92, 93, 94, 95, 96

Consumo de alimentos 105

Contaminação 43, 44, 45, 47, 49, 72, 73, 74, 78, 117, 135, 179, 191, 193, 195, 197

Coronavac (sinovac) 28, 29

Cuidados individuais e coletivos $67,69,73$

Cuidados preventivos ao covid-19 115

D

Depressão 78, 81, 82, 83, 87, 91, 92, 94, 95, 110, 172, 190, 192, 193, 194, 197, 207, 208, 209

Desemprego 21, 99, 103, 153

Desenvolvimento do câncer 177, 185

Desigualdade em saúde 163

Desinformação 29, 208 
Diabetes 21, 56, 57, 95, 105, 106, 107, 108, 114, 116, 117, 118, 119, 120, 181, 183, 186

Direito humano à alimentação adequada 103, 260

Disseminação da informação 67,73

Distanciamento social 25, 48, 55, 61, 68, 73, 90, 95, 126, 130, 192, 195, 202, 279

Distúrbios psicológicos 190

Doença infecciosa 53, 125, 133, 134, 140, 141, 151, 152

Doença multifatorial 250

Doenças 21, 24, 28, 29, 43, 54, 56, 57, 59, 69, 91, 95, 106, 107, 108, 115, 116, 117, 119, 120, 129, 133, 137, 140, 142, $149,161,163,164,167,171,172,173,177,185,186,193,194,195,207,210,217,222,223,227,250,271$, $272,273,278,279$

Doenças crônicas 105, 171, 218, 221, 227, 273

Doenças negligenciadas 140, 142

\section{$\mathbf{E}$}

Educação em saúde 19, 24, 43, 46, 48, 50, 67, 69, 73, 105, 108, 133, 134, 135, 136, 137, 145, 179, 185, 215, 216, 217, $227,228,238,240,248,271,272,274$

Efeitos colaterais $28,30,31,32,33$

Enfermagem 50, 56, 57, 58, 60, 61, 88, 89, 122, 149, 161, 176, 177, 178, 180, 188, 227, 240, 280

Enfermidade epidêmica 238

Enfrentamento da covid-19 18, 19, 20, 23, 24, 25

Epidemiologia 114, 140, 148, 149, 160, 161, 242, 248

Equipamentos de proteção 24, 43, 47, 78, 171, 197

Equipes multiprofissionais $52,54,55,56,59,60,61,62$

Estratégia saúde da família (esf) 18, 19, 23, 54

Estratégias nutricionais 105

Estresse 77, 78, 81, 82, 83, 86, 87, 88, 89, 94, 172, 190, 192, 193, 197, 206, 207, 209, 210, 211

Etiologia autoimune 250

Evidências científicas 29, 52, 56, 57, 59, 61, 62, 63, 76, 79, 99, 101, 190

F

Feiras agroecológicas 260, 261, 264

Ferramenta tecnológica de saúde 215

Fortalecimento da saúde púbica 67

Frequência cardíaca 230

G

Gestação 52, 53, 54, 57, 58, 59, 61, 62, 63, 224

Glândulas exócrinas 250, 251, 252, 253

Guia alimentar 105, 107, 108, 112, 113

$\mathbf{H}$

Hábitos alimentares 90, 92, 94, 95, 111, 112

Hanseníase 124, 125, 126, 127, 128, 130, 131, 133, 134, 135, 136, 137, 138, 139, 140, 141, 142, 143, 144, 145, 146, $147,148,149$

Herança genética 115

Hipertensão 57, 95, 105, 107, 108, 114, 115, 116, 118, 119, 120, 121, 181, 183, 186, 228, 229, 274, 276,278 
Hiposecreção lacrimal 250

I

Idosos 19, 21, 23, 54, 105, 108, 134, 137

Impacto da pandemia 99

Imunização 28, 29, 30, 35, 38, 179, 183, 186

Inclusão 20, 22, 52, 55, 56, 58, 75, 77, 79, 101, 163, 165, 167, 215, 226, 268

Infecções 28, 29, 38, 43, 44, 48, 57, 59, 60, 78, 165, 172, 180, 227, 251

Infecções respiratórias virais $43,44,48$

Informação de qualidade 271, 279

Informação em saúde 67,69

Informação sobre a hanseníase 124

Instabilidade econômica 99, 103

Instrumento de prevenção 43

Intervenção psicológica 202

Isolamento $23,48,55,60,62,81,90,91,92,94,100,142,164,177,179,190,191,192,193,194,195,196,197,198$, $199,203,204,205,207,208,210,242,261$

Isolamento social 23, 26, 62, 90, 92, 94, 100, 142, 164, 177, 179, 190, 191, 192, 194, 195, 196, 197, 198, 203, 205, 207, 208, 210, 242

$\mathbf{J}$

Janssen (johnson \& johnson) 28, 29, 30

$\mathbf{L}$

Lesões cutâneas 140, 143

Linha de frente 76, 78, 79, 81, 84, 86, 87, 88, 171, 195, 197, 202, 206, 207, 208, 210

M

Má qualidade do sono 77,87

Máscaras faciais 43

Material digital 43, 46

Medição biométrica 230

Medidas de isolamento 26, 28, 194

Medidores de glicose 230

Metodologia pico (problema, intervenção, contexto, resultado) 76, 79, 99, 101

Monitores do centro de ciências da saúde (ccs) 238

Monitoria 238, 239, 240, 241, 242, 243, 244, 246

Morbidade 115, 119

Mortalidade materna 163, 171, 172

Mudanças nos estilos de vida 90, 92, 93

Mycobacterium leprae 135, 140, 141, 146

N

Níveis socioeconômicos 43, 46

Novas modalidades de interações 238

Novo coronavírus sars-cov-2 18, 19 
Olho seco 250, 251, 252, 253, 254, 255, 256, 257, 258

Organização mundial de saúde (oms) 19, 52, 53, 54, 68, 92, 191

Organização pan-americana de saúde (opas) 52, 54

Órgãos oficiais de saúde 238, 247

Outubro rosa $177,178,179,184,185$

$\mathbf{P}$

Padrões dietéticos 115

Pandemia 18, 19, 20, 21, 23, 24, 25, 26, 28, 31, 38, 45, 47, 48, 53, 55, 68, 69, 73, 75, 76, 78, 79, 81, 82, 84, 86, 87, 88, $89,90,91,92,93,94,95,96,99,100,101,102,103,104,117,118,119,120,124,126,130,131,133,135,136$, $137,142,152,153,160,161,162,163,164,165,168,169,170,171,172,173,174,177,179,180,186,187$, 190, 191, 192, 193, 194, 195, 196, 197, 198, 199, 200, 203, 204, 205, 206, 207, 208, 210, 211, 212, 213, 238, $239,240,241,242,244,247,248,271,272,279,280$

Perfil alimentar 105

Perfil clínico 160, 177, 183, 185

Pfizer (pfizer e biontech) 28, 30

Políticas públicas de saúde 18, 24, 173, 204

Portadores de síndrome metabólica 115

Prática oftalmológica 250

Práticas do autocuidado 178,186

Pressão arterial 115,230

Prevenção 29, 33, 72, 170, 187, 221

Primeiros socorros 215, 218, 219, 225, 227, 273

Princípio ativo 28, 30, 33

Produção de alimentos 260

Profissionais de psicologia 202

Profissionais de saúde 18, 22, 24, 54, 56, 76, 78, 79, 81, 82, 84, 85, 86, 87, 89, 107, 125, 138, 151, 160, 170, 185, 194, $195,197,198,202,206,207,208,238$

Programa nacional de alimentação escolar (pnae) 99, 265

Promoção em saúde 43, 45, 271, 278

Propagação do vírus $43,47,48,67,69$

Q

Qualidade de vida 22, 47, 82, 106, 112, 155, 217, 226, 244, 250, 251, 254, 272, 278

Quarentena 90, 100, 121, 126, 170, 172, 190, 191, 192, 193, 194, 195, 199, 202, 206

$\mathbf{R}$

Recurso tecnológico 230

Redes sociais 43, 45, 46, 49, 133, 137, 194, 271, 272, 274, 278, 279

Responsabilidade individual e coletiva 18, 24

Restrição social 90, 92, 93 
Sars-cov- $20,21,29,30,31,32,33,34,35,38,39,40,45,49,53,56,57,58,59,60,65,88,120,163,164,165,168$, $173,191,199,238,239,240,242,243,247,248,279$

Saúde da família 18, 19, 20, 22, 23, 24, 25, 26, 52, 54, 55, 62, 64, 125, 135, 179, 187

Saúde da mulher 163, 166, 173

Saúde de maneira remota 133

Saúde dos profissionais $76,79,81,86,89$

Saúde dos trabalhadores $77,86,87$

Saúde integral 52, 163, 165, 167, 171

Saúde mental 78, 83, 88, 90, 93, 94, 95, 96, 172, 179, 190, 191, 192, 193, 194, 195, 196, 197, 198, 202, 203, 204, 206, $207,208,210,212,222,227,279$

Saúde psicológica 77,87

Saúde pública $6,20,28,38,69,73,82,120,125,134,139,141,148,149,160,168,169,170,171,172,177,179,197$, $199,208,211,238,240,242,247$

Secura ocular 250, 251, 257

Sedentarismo 94, 107, 115, 179

Segurança alimentar e nutricional 99, 100, 101, 102, 103, 104

Segurança alimentar nutricional (san) 99

Serviço de saúde 71, 115, 120, 180

Síndrome de sjögren (ss) 250, 251

Síndrome metabólica 115, 116, 117, 118, 119, 120

Sistema imunológico 58, 115, 117, 120

Sistema nacional de agravos de notificação (sinan) 140, 143

Situação de vulnerabilidade 90, 94, 95, 103

Superfície ocular 250, 254, 258

T

Tecnologia 66, 67, 69, 70, 137, 211, 231, 244

Tecnologias de informação e comunicação 208, 230

Teleatendimento 202, 204

Telemedicina 133, 170, 230, 232

Telessaúde 230, 236

Tempos pandêmicos 271

Terapêutica multidisciplinar 190

Teste de schirmer 250, 255

Testes oftalmológicos 250

Transmissão de informações 271, 279

Transtorno de ansiedade generalizada 190, 192

Tuberculose (tb) 151,152

$\mathbf{U}$

Unidade de saúde da família (usf) 52, 55

Uso das máscaras 43, 45, 48 
Vacina 28, 31, 32, 33, 34, 37, 39, 40, 70, 72, 159, 161, 183, 184, 186, 221

Variantes $28,30,31,34,35,38,49,78$

Vídeos educativos 215, 226

Violência contra a mulher $163,169,170,173,174$

$\mathbf{X}$

Xeroftalmia 250,251 


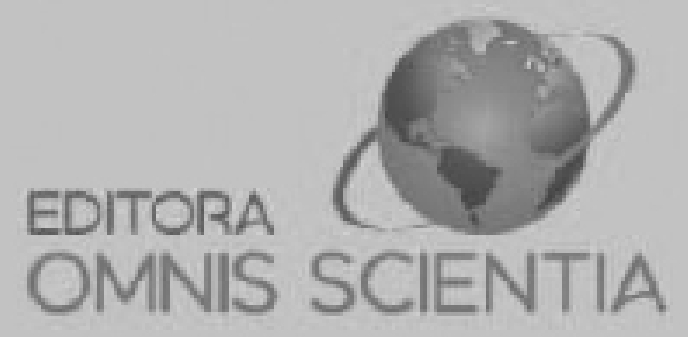

editoraomnisscientia@gmail.com M https://editoraomnisscientia.com.br/ @editora_omnis_scientia (0) https://wwww.facebook.com/omnis.scientia.9 $\boldsymbol{\oplus}$ +55 (87) 9656-3565 (C) 


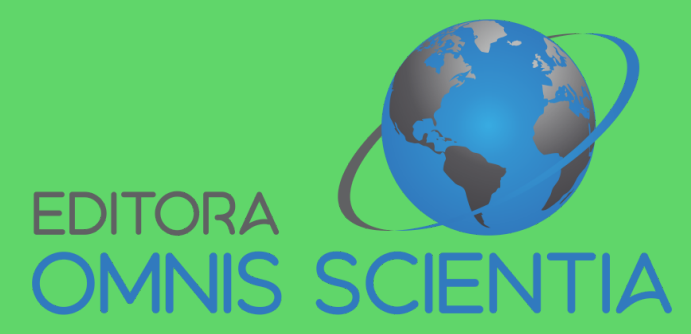

editoraomnisscientia@gmail.com M https://editoraomnisscientia.com.br/ (-) @editora_omnis_scientia (0) https://www.facebook.com/omnis.scientia.9 $\subsetneq$ +55 (87) 9656-3565 @ 